\title{
Construção e validação de folheto para cuidadores de idosos sobre prevenção da COVID-19
}

Flávia de Oliveira ${ }^{1}$, ${ }^{(}$Hemelly Nogueira Guimaraes $^{1}{ }^{(0)}$, Greyce Hellen Rabelo Cezar $^{1}{ }^{(\mathbb{D})}$, Rayssa Nogueira Rodrigues Machado ${ }^{(\mathbb{D}}$, Aline Carrilho Menezes ${ }^{\mathbb{( B}}$, Diego Dias de Araújo ${ }^{(\mathbb{D}}$, Silmara Nunes Andrade $^{2}$, Danilo Donizetti Trevisan ${ }^{1}$

\section{RESUMO}

Fundamentos: Como ferramenta de cuidado da população idosa, é importante que cuidadores tenham conhecimento sobre a prevenção da COVID-19 a fim de minimizar a transmissão do SARS-CoV-2. Objetivo: Descrever o processo de construção e validação de conteúdo de folheto educativo para cuidadores de idosos sobre medidas de prevenção da COVID-19. Métodos: Estudo metodológico desenvolvido em três etapas. A primeira etapa corresponde à revisão de literatura; a segunda envolveu a construção do folheto e a última etapa a validação de conteúdo por um comitê de 23 especialistas que julgaram os objetivos, estrutura/apresentação e relevância do folheto a partir de uma escala validada tipo Likert de 3 pontos. Itens com opiniões positivas por mais de $80,0 \%$ foram considerados adequados. Resultados: $O$ folheto foi elaborado contendo seis páginas, com informações sobre o novo coronavírus, formas de transmissão, sinais e sintomas, proteção da doença, alteração da rotina dos cuidados diários, importância da higienização das mãos, superfícies e alimentos; uso correto de máscara; cuidados específicos ao cuidador ou familiar; saúde emocional e como proceder em caso de suspeita da COVID-19. Apenas uma rodada de avaliação foi necessária; o índice de validade de conteúdo foi de 1,0 e a concordância entre juízes foi estatisticamente significante em todos os domínios da escala $(p<0,006)$. Conclusão: Este estudo disponibiliza um folheto educativo com conteúdo validado, configurando-se um componente passível para aprimorar o conhecimento de cuidadores a respeito das medidas de prevenção contra a COVID-19 em idosos.

Palavras-chave: Infecções por coronavírus, Cuidadores, Idoso, Materiais educativos e de divulgação, Estudos de validação.

1. Universidade Federal de São João Del Rei, Divinópolis, (MG), Brasil.

2. Universidade do Estado de Minas Gerais, Divinópolis, (MG), Brasil.

3. Universidade Estadual de Montes Claros, Montes Claros, (MG), Brasil. 


\section{INTRODUÇÃO}

$\mathrm{Na}$ atualidade, a saúde pública enfrenta um novo desafio relacionado à pandemia da COVID-19 causada pelo coronavírus 2 da síndrome respiratória aguda grave (SARS-CoV-2). Esta doença pode afetar pessoas de todas as idades e de diferentes maneiras. Dados da Organização Mundial da Saúde (OMS) confirmaram mais de 399,5 milhões de pessoas acometidas pela COVID-19 e ultrapassaram a marca de 5,7 milhões de mortes em todo o mundo, caracterizando uma doença de rápida contaminação e disseminação. No Brasil, mais de 630 mil vítimas evoluíram a óbito, com taxa de letalidade de aproximadamente $2,5 \% \cdot{ }^{1,2}$

A maioria das pessoas infectadas são assintomáticas ou apresentam sintomas leves a moderados da doença como febre (temperatura $\geq$ $37,8^{\circ} \mathrm{C}$ ), calafrios, odinofagia, cefaleia, mialgia, tosse seca, coriza, distúrbios olfativos (anosmia), gustativos (ageusia) e, com isso, não necessitam de hospitalização. Por outro lado, a infecção também tem o potencial de acarretar sintomas graves como dispneia e desconforto respiratório e evoluir para Síndrome Respiratória Aguda Grave (SRAG) com necessidade de internação em unidade de terapia intensiva e, muitas vezes, resultando em morte. ${ }^{3,4}$ Ademais, pelo menos $10 \%$ das pessoas acometidas podem manter sintomas persistentes ou apresentar complicações tardias do início da doença, incluindo fadiga, mal-estar, tosse seca, alterações cardiovasculares, neurológicas, psiquiátricas e metabólicas. ${ }^{5}$

Neste sentido, diferentes estratégias terapêuticas têm sido desenvolvidas e pesquisadas para prevenção, tratamento e/ou controle da disseminação da doença. Medicamentos foram e estão sendo testados por diversos grupos de pesquisadores em todo o mundo quanto aos seus respectivos potenciais contra o novo coronavírus, entretanto, ainda há uma ampla discussão na literatura sobre a eficácia de cada um deles..$^{6-9}$ Até o momento, é consenso na literatura, como medida de prevenção, a administração de vacinas; ainda que nenhuma delas apresente $100 \%$ de eficácia, as mesmas têm o potencial de contribuir na redução da severidade da doença caso haja exposição ao vírus SARS-CoV-2. ${ }^{10-12}$

Diante desse cenário, justifica-se a realização e a manutenção das medidas não farmacológicas para prevenção da doença, principalmente relacionado aos grupos vulneráveis, como idosos e pessoas com doenças graves e/ou com comorbidades subjacentes. ${ }^{2}$ Idosos tendem a apresentar limitações na autonomia e independência, características do processo de envelhecimento, ocasionando, muitas vezes, a necessidade de um cuidador, membro ou não da família, para auxiliar ou executar o cuidado relacionado às atividades de vida diárias. ${ }^{13}$ Assim, como ferramenta de prevenção da COVID-19 em idosos, é importante que os cuidadores tenham conhecimento satisfatório sobre as maneiras de prevenir e minimizar o risco de contaminação e transmissão do SARS-CoV-2.

As medidas de prevenção da COVID-19 são ações de baixo custo e de alta efetividade que se referem principalmente ao isolamento domiciliar, ao distanciamento físico, ao uso de máscaras, à higiene adequada das mãos e a etiqueta respiratória. ${ }^{14}$ Entretanto, observa-se que parte da população não adere totalmente a essas medidas. Considera-se que o envolvimento para a adoção consciente das medidas de precaução frente à COVID-19 exige uma mudança de comportamentos individual e coletivo bem como imediato e preciso. ${ }^{15}$ As abordagens para atingir esse propósito devem envolver a educação em saúde por serem importantes para o processo de mudança social e empoderamento das pessoas. ${ }^{16}$

Práticas de educação em saúde associadas a recursos tecnológicos favorecem a mediação de processos de ensinar e aprender no cuidado. Estas tecnologias viabilizam a disseminação da informação, facilitam a compreensão e ampliam as facetas da educação formal. ${ }^{17}$ Materiais educativos, sejam impressos ou digitais, tais como manuais, folhetos e cartilhas podem contribuir positivamente para orientação e informação do cuidado com o idoso bem como para a tomada de decisão do cuidador. ${ }^{18}$ Em especial, os folhetos educativos podem ser capazes de nortear ações de cuidados em domicílio e de contribuir para o desenvolvimento da capacidade de interferência do receptor da informação em processos decisórios. ${ }^{19}$

Para nosso conhecimento, após a realização de buscas nas bases de dados, não foram identificados materiais educativos validados direcionados aos cuidadores de idosos com foco na prevenção da COVID-19 e com linguagem de fácil compreensão para este público-alvo. Assim, este estudo objetivou descrever o processo de construção e validação de conteúdo de folheto educativo para cuidadores de idosos sobre medidas de prevenção da COVID-19. 


\section{MÉTODOS}

\section{Desenho, local do estudo e período}

Trata-se de um estudo metodológico, com abordagem quantitativa, envolvendo a construção e validação de folheto educativo direcionado para cuidadores de idosos sobre a prevenção da COVID-19, realizado entre maio e agosto de 2020 , em um município da região centro-oeste de Minas Gerais, Brasil. Para a construção do folheto, as seguintes etapas foram estabelecidas: 1- revisão integrativa; 2 - construção do folheto educativo junto ao designer gráfico para auxílio do processo de desenvolvimento da tecnologia educativa e 3 - validação de conteúdo por um comitê de especialistas. ${ }^{20}$

O referencial teórico utilizado para o desenvolvimento do folheto educativo baseou-se no Modelo de Crenças em Saúde (Health Belief Model HBM) que considera os fatores que podem influenciar o comportamento em saúde de uma determinada população. ${ }^{21}$ Estes fatores são: percepção para 0 fato de se encontrar susceptível a alguma situação de risco à saúde (contemplado no folheto com conteúdo acerca de uma maior susceptibilidade dos idosos serem contaminados pela COVID-19); percepção para a gravidade de tal situação (abordado no folheto por conteúdo referente às consequências da COVID-19; estas muitas vezes graves ou fatais); percepção para os benefícios de realizar ações que diminuam o risco (contemplados no folheto nas formas de como prevenir a transmissão da COVID-19 para os idosos); a percepção para as barreiras acerca da realização de tais ações (abordado no material educativo em conteúdo que descrevia possíveis dificuldades do cotidiano para realizar as medidas de prevenção para a COVID-19)..$^{21}$

\section{Levantamento bibliográfico}

A revisão integrativa foi realizada em junho de 2020 e as seguintes bibliotecas/bases de dados foram consultadas: PubMed, BVS e SCOPUS. Os descritores utilizados foram Covid-19, Coronavírus, Cuidadores, Idosos e as estratégias de busca estruturadas foram: Pubmed - ((("COVID-19" [Supplementary Concept])) OR "Coronavirus"[Mesh]) AND "Caregivers"[Mesh]) AND ("Aged"[Mesh] OR "Health Services for the Aged"[Mesh]); BVS - (tw:(covid-19)) OR (tw:(coronavirus)) AND (tw:(Cuidadores)) AND (tw:(Idoso)) OR (tw:(Saúde do Idoso)); SCOPUS - (
TITLE-ABS-KEY ( "COVID-19") OR TITLE-ABS-KEY ( coronavirus ) AND TITLE-ABS-KEY (caregivers) AND TITLE-ABS-KEY ( aged ) OR TITLE-ABS-KEY ( "Health Services for the Aged") ).

Os critérios de inclusão foram: artigos, guidelines ou recomendações de órgãos nacionais e internacionais disponibilizados nos idiomas inglês, espanhol ou português sem período de tempo estipulado. Foram excluídos materiais repetidos e que não abordavam a temática do estudo.

Foram identificados 15 estudos no PubMed, 108 na BVS e 54 na SCOPUS. Após a leitura dos resumos e textos na íntegra, foram selecionadas oito publicações (artigos e outros documentos de pesquisa) para leitura reflexiva e síntese dos pontos mais relevantes que nortearam a elaboração do conteúdo do folheto educativo. Nenhum material educativo foi identificado que visasse orientações sobre prevenção da COVID-19 para cuidadores de idosos. Foram coletadas informações referentes ao título, ano de publicação, país, idioma, objetivo, método, resultados, conclusão e nível de evidência. Foram incluídas também informações presentes nos protocolos de prevenção da COVID-19 disponibilizados pela OMS, Ministério da Saúde (MS) e Agência Nacional de Vigilância Sanitária (ANVISA).

\section{Construção do folheto educativo}

Após estabelecer o conteúdo pertinente para o folheto educativo, iniciou-se o processo de construção fundamentado em três aspectos: linguagem, ilustração e layout/design, conforme recomendação para a elaboração de materiais educativos em saúde. ${ }^{20}$ Os textos foram elaborados com linguagem objetiva e direcionada ao público-alvo e as imagens foram desenhadas a mão por uma das autoras.

Em seguida, o material (composto por textos e as imagens digitalizadas) foi entregue para um profissional especialista na área de designer para o aprimoramento das ilustrações bem como diagramação de acordo recomendações para elaboração de materiais educativos. ${ }^{22}$ As ilustrações foram criadas e desenvolvidas pelos softwares Krita e Sketchbook e, posteriormente, finalizadas no Adobe Illustrator.

\section{Validação de conteúdo pelo comitê de especialistas}

Após a construção do folheto, o processo de validação de conteúdo foi realizado por um comitê de 
especialistas. O tamanho da amostra para a seleção dos especialistas foi calculado de acordo com a seguinte fórmula: $n=Z a^{2} . P(1-P) / e^{2}$, sendo $Z a$ (nível de confiança) $=95 \%, P$ (proporção de concordância mínima dos especialistas) $=85 \%$, e (diferença aceitável do esperado) $=15 \%$; resultando em uma amostra mínima, de 22 participantes. ${ }^{23}$

Para a seleção dos especialistas, os seguintes critérios de inclusão foram estabelecidos ${ }^{24}$ : possuir expertise sobre a temática saúde do idoso, infectologia ou saúde pública; conhecimento/ habilidade adquiridos por experiência profissional mínima de um ano (assistência, ensino ou pesquisa) e ter experiência no desenvolvimento de tecnologias em saúde ou artigos publicados sobre a temática.

Assim, a busca pelos especialistas ocorreu a partir da amostragem do tipo bola de neve, ou seja, foi solicitado a cada participante que indicassem outros profissionais com perfil elegível para participação no estudo. Para a coleta de dados, cada especialista recebeu, via e-mail, a carta convite explicando o objetivo do estudo, o Termo de Consentimento Livre e Esclarecido (TCLE), o roteiro de caracterização de perfil, o Instrumento de Validação de Conteúdo Educativo em Saúde $(\text { IVCES })^{25}$, e o folheto propriamente dito. Após o envio de e-mails para 30 potenciais participantes, 23 enviaram a concordância em participar do estudo. 0 prazo inicial estipulado para o retorno da avaliação foi de 15 dias, entretanto, o mesmo necessitou ser prorrogado por mais 15 dias para atingir o número mínimo de avaliações.

O IVCES é composto por dezoito itens divididos em três domínios: 1) Objetivos: propósitos, metas ou finalidades; 2) Estrutura/Apresentação: organização, estrutura, estratégia, coerência e suficiência; 3) Relevância: significância, impacto, motivação e interesse. A partir de uma escala tipo Likert, as opções de respostas variavam de 0 a 2, sendo $0=$ discordo, 1 = concordo parcialmente e 2 = concordo totalmente; um coeficiente de correlação intraclasse final de 0,877 foi reportado, indicando consistência interna satisfatória. ${ }^{25}$

Ademais, ao final do instrumento constava um espaço aberto para comentários e sugestões. Todas as sugestões enviadas pelos especialistas foram organizadas e analisadas conforme as variáveis do instrumento, as quais foram devidamente realizadas.

\section{Análise dos dados}

Os dados foram analisados com auxílio do software Statistical Package for the Social Sciences (SPSS) versão 23. Realizou-se a análise descritiva das variáveis de caracterização dos especialistas. Para verificar a validade de conteúdo do folheto, calculouse o Índice de Validade de Conteúdo (IVC) da seguinte forma: I-CVI (Item-Level Content Validity Index) que corresponde à concordância dos avaliadores para cada item e o IVC global - somatório de todos os I-CVI, dividido pela quantidade de avaliadores. O ponto de corte mínimo de concordância para que o item fosse considerado válido foi de $0,80 .{ }^{26}$ Por fim, o teste binomial, com nível de significância de $5 \%$ foi realizado para verificar, estatisticamente, se a concordância entre os especialistas foi igual ou superior a $80 \%$.

\section{Aspectos éticos}

O estudo foi aprovado pelo Comitê de Ética em Pesquisa local (CAAE 30659520.0.0000.5545) e atendeu à resolução 466/2012 do Conselho Nacional de Saúde bem como a Declaração de Helsinque da Associação Médica Mundial.

\section{RESULTADOS}

O folheto foi intitulado "Promoção de cuidados ao idoso em época de COVID-19" e composto por seis páginas (capa, páginas de apresentação sobre a temática, referências e ficha técnica). O conteúdo do folheto foi apresentado com a seguinte sequência: apresentação sobre o novo coronavírus; forma de transmissão; sinais e sintomas; formas de proteção da doença; alteração da rotina dos cuidados diários; importância da higienização das mãos, superfícies e alimentos; uso correto de máscara; cuidados específicos ao cuidador ou familiar; saúde emocional do idoso e como proceder em caso de suspeita da COVID-19. As ilustrações das páginas do folheto são apresentadas nas Figuras $1 \mathrm{~A}, 1 \mathrm{~B}$ e $1 \mathrm{C}$. $\mathrm{O}$ acesso na íntegra pode ser realizado pelo download do material em formato PDF no link: https://drive.google.com/file/d/15mubJQWVMy8xr2qiZIVKbO1gOSOBB-P/view?usp=sharing. 


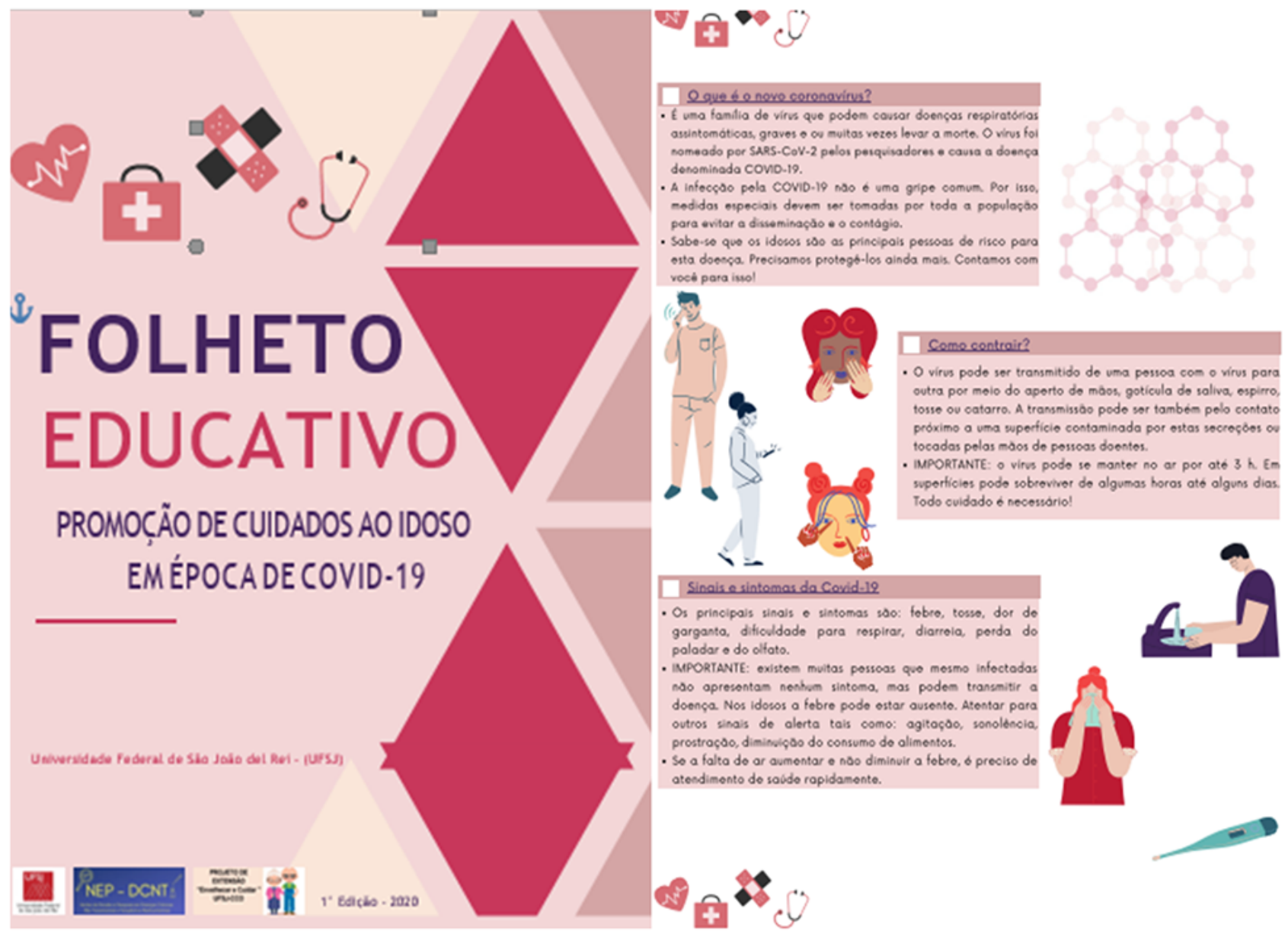

Figura 1A. Páginas 1 e 2 do folheto educativo. Divinópolis, Minas Gerais, 2020.

A validação de conteúdo foi realizada por 23 especialistas, sendo dois $(8,7 \%)$ médicos, uma $(4,3 \%)$, fisioterapeuta e $20(87 \%)$ enfermeiros. Quanto à titulação, quatro $(17,3 \%)$ eram especialistas, nove $(39,1 \%)$ mestres e dez $(43,5 \%)$ doutores. Em relação à ocupação atual, três (13\%) eram estudantes de curso de pós-graduação (mestrado ou doutorado), dez $(43,5 \%)$ atuavam como profissionais assistenciais, dois $(8,7 \%)$ docentes do curso de graduação em medicina e oito $(34,8)$ docentes do curso de graduação em enfermagem. A maioria $(56,5 \%, n=13)$ atuava em instituições de ensino superior, seguidos de unidades básicas de saúde $(26,1 \%, n=6)$. $O$ tempo médio de formação foi de 11,5 anos $( \pm 8,3)$.

Os 18 itens avaliados pelo IVCES referentes aos objetivos, estrutura/apresentação e relevância encontram-se detalhados na Tabela 1. Nenhum item recebeu avaliação de discordância e alcançou 1,0 ponto no I-CVI e, consequentemente, IVC global de 1,0 . O teste binomial evidenciou significância estatística $(p=0,006)$ na concordância igual ou superior a 0,80 entre os juízes em todos os itens avaliados. Diante desse resultado, apenas uma rodada na validação com os juízes foi necessária.

Entretanto, mesmo com elevados níveis de concordância e de um IVC global de 1,0, alguns juízes fizeram sugestões para aprimorar as informações do folheto. No item "Sinais e sintomas de COVID-19", os especialistas sugeriram incluir a informação de que nos idosos a febre nem sempre está presente, bem como observar sinais de agitação, prostração, sonolência e ingesta alimentar diminuída. A frase escrita anteriormente como "Dificuldade para respirar, começando com um cansaço leve podendo progredir rapidamente, levando à insuficiência respiratória grave", foi reelaborada como "Dificuldade ao respirar, representada por um cansaço leve, pode progredir rapidamente para uma insuficiência respiratória grave. Nessa situação é recomendada a ida imediata à emergência para atendimento". 

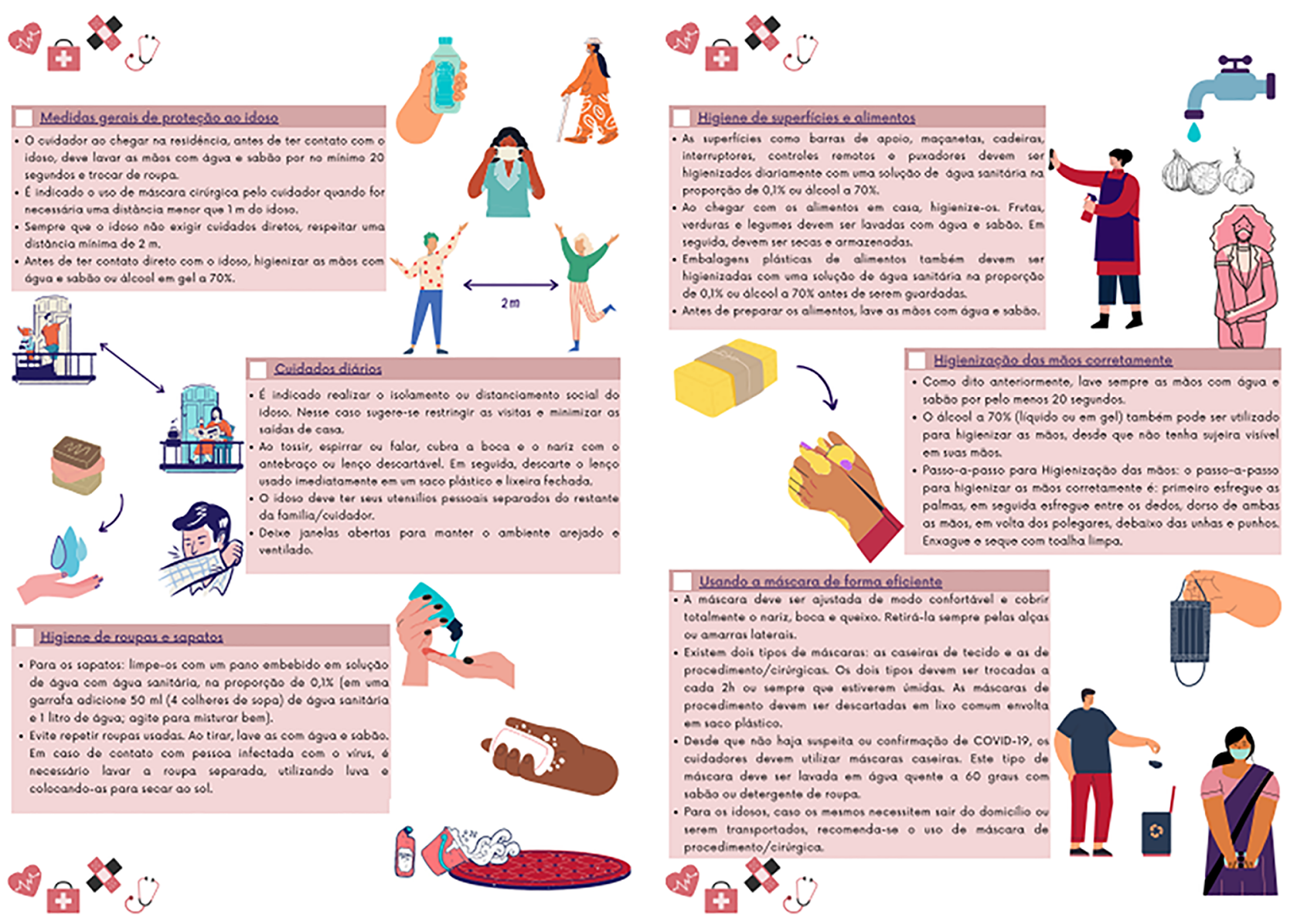

Figura 1B. Páginas 3 e 4 do folheto educativo. Divinópolis, Minas Gerais, 2020.

Outra frase reescrita foi "É indicado restringir as visitas aos idosos" para "É indicado realizar o isolamento ou distanciamento social do idoso. Nesse caso sugere-se restringir as visitas e minimizar as saídas de casa". Foi ajustado também informações sobre a limpeza e desinfecção em geral e o uso de máscaras. A frase "A limpeza e desinfecção de superfícies diversas como maçanetas, bancadas, mesas, cadeiras, embalagens deve ser realizada com álcool a $70 \%$ ou hipoclorito de sódio (água sanitária)" foi reescrita para "As superfícies como barras de apoio, maçanetas, cadeiras, interruptores, controles remotos e puxadores devem ser higienizados diariamente com uma solução de água sanitária na proporção de $0,1 \%$ ou álcool a $70 \% "$. Destaca-se que a proporção de diluição já havia sido explicada anteriormente no folheto.

Em relação ao uso correto de máscaras, foi explicitado no folheto qual o tipo adequado de máscara indicada para o cuidador e o idoso e em qual ocasião deveriam utilizar. Inicialmente, havia a indicação de reuso somente após lavagem, secagem e passagem de ferro. Essa informação foi ajustada para "as máscaras de tecido devem ser lavadas em água quente a $60^{\circ} \mathrm{C}$ com sabão ou detergente de roupa". Para os idosos, caso os mesmos necessitem sair do domicílio ou serem transportados, recomendase o uso de máscara procedimento/cirúrgica. Ao final de todas as correções o folheto passou por uma corretora da língua portuguesa do Brasil.

\section{DISCUSSÃO}

O presente estudo objetivou construir e validar o conteúdo de um folheto educativo para cuidadores sobre a prevenção da COVID-19 em idosos. Por unanimidade do comitê de especialistas, todos os itens avaliados pelo IVCES receberam avaliação de concordância parcial ou total. O processo de validação com avaliação de itens referentes ao objetivo, estrutura/apresentação e relevância é importante para que os materiais 


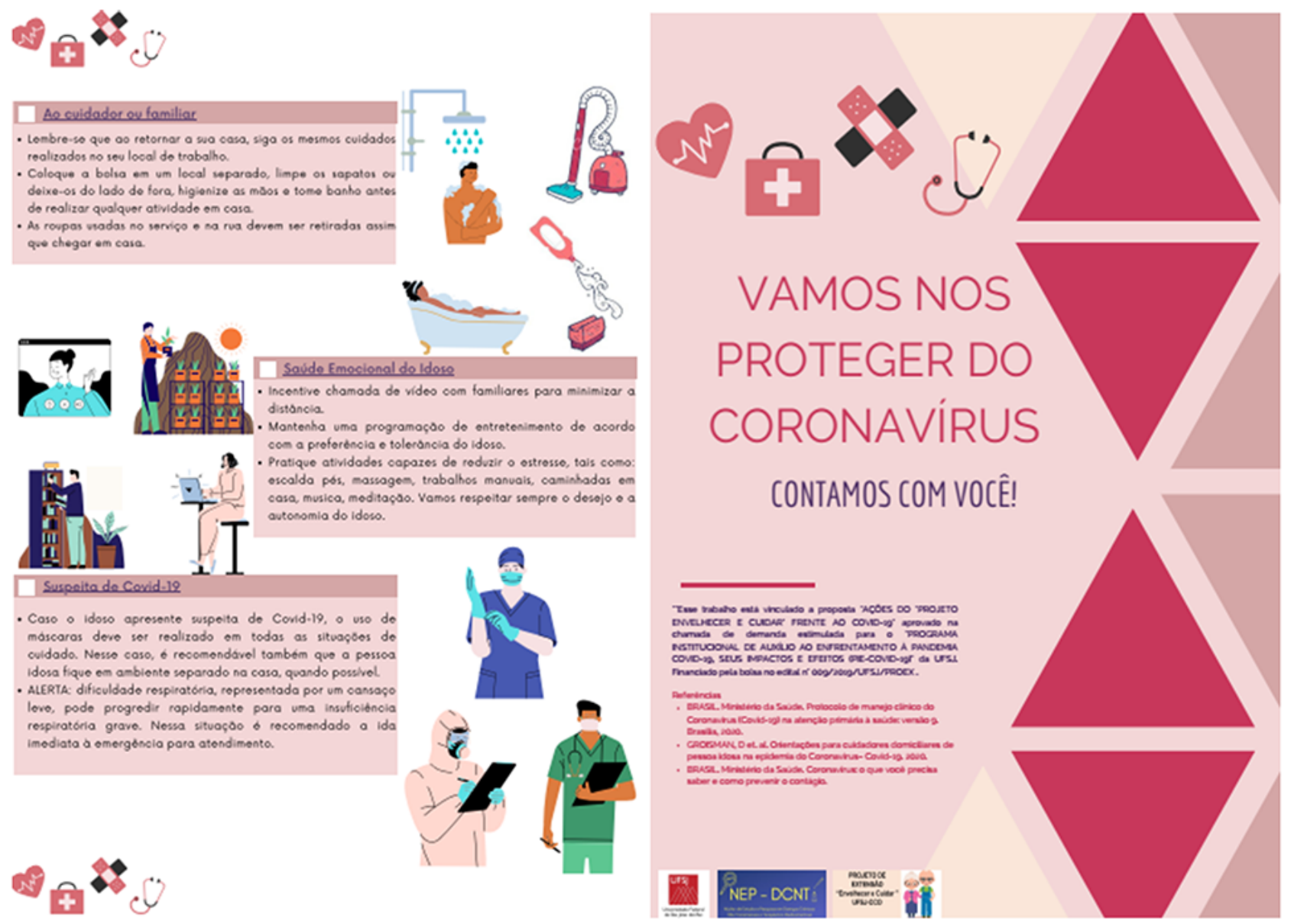

Figura 1C. Páginas 5 e 6 do folheto educativo. Divinópolis, Minas Gerais, 2020.

educativos apresentem, de forma intuitiva, o universo de informações técnico-científicas e exclua os elementos desnecessários à transferência de conhecimento. ${ }^{27}$ Destaca-se, entre os itens avaliados, a abordagem do tema por meio das ilustrações. Estudo aponta que os materiais com imagens são visualmente atraentes e preferidos, o que os torna mais propensos a serem lidos. ${ }^{28}$

Especificamente, acerca das tecnologias de educação em saúde utilizadas no cuidado ao idoso, resultados de revisão integrativa descrevem que $o$ uso de tecnologias em saúde, entre eles 0 folheto educativo, estabelece condições adequadas e inovadoras para um cuidado satisfatório. ${ }^{29} \mathrm{O}$ emprego de materiais educativos tem a capacidade de estreitar a comunicação da assistência em saúde ao considerar as características da população-alvo.

Embora os cuidadores sejam reconhecidos como parte integrante do sistema de atenção ao idoso, a elevada média de idade juntamente com o baixo nível de escolaridade podem ser barreiras para desempenhar as atividades de cuidado, como administrar medicamentos, receber e transmitir orientações de profissionais da saúde e informar-se sobre o recebimento de recursos públicos (utilização do Benefício de Prestação continuada, por exemplo). ${ }^{30}$ O cuidado informal, não remunerado, normalmente é feito pelo cônjuge, irmão, amigo, vizinho ${ }^{31}$, ou seja, por pessoas que enfrentam dificuldades semelhantes ao receptor do cuidado. Além disso, a sobrecarga imposta aos cuidadores os coloca em risco de problemas de saúde. Nesta direção, um estudo transversal apresentou que a carga horária de trabalho de cuidadores informais é quase o triplo quando comparados com a de cuidadores formais $\left(19,8\right.$ e 7,5 horas diárias, respectivamente) ${ }^{32}$

Nesse contexto, diversas preocupações podem surgir pelo fato do acesso aos serviços de suporte serem escassos. O cuidador frequentemente se depara com situações inesperadas e, como consequência, necessita de aconselhamento e de orientações no que tange o conhecimento sobre 
Tabela 1. Concordância dos especialistas em relação aos objetivos, estrutura, apresentação e relevância do folheto. Divinópolis, MG, Brasil, 2020. $(n=23)$

\begin{tabular}{|c|c|c|c|}
\hline \multirow{2}{*}{ Itens avaliados } & Concordo totalmente & Concordo & \multirow{2}{*}{$\mathrm{I}-\mathrm{CVI} *$} \\
\hline & $\mathrm{n}(\%)$ & $\mathrm{n}(\%)$ & \\
\hline 1. & Objetivos & & \\
\hline 1.1 Contempla o tema proposto & $22(95,7)$ & $1(4,3)$ & 1,00 \\
\hline $\begin{array}{l}1.2 \text { Adequado ao processo de ensino- } \\
\text { aprendizagem }\end{array}$ & $20(87,0)$ & $3(13,0)$ & 1,00 \\
\hline 1.3 Esclarece dúvidas sobre o tema & $22(95,7)$ & $1(4,3)$ & 1,00 \\
\hline 1.4 Proporciona reflexão sobre o tema & $22(95,7)$ & $1(4,3)$ & 1,00 \\
\hline 1.5 Incentiva mudança de comportamento & $19(82,6)$ & $4(17,4)$ & 1,00 \\
\hline 2. & $\begin{array}{c}\text { Estrutura e } \\
\text { Apresentação }\end{array}$ & & \\
\hline 2.1 Linguagem adequada ao público-alvo & $16(69,6)$ & $7(30,4)$ & 1,00 \\
\hline $\begin{array}{l}2.2 \text { Linguagem apropriada ao material } \\
\text { educativo }\end{array}$ & $19(82,6)$ & $4(17,4)$ & 1,00 \\
\hline 2.3 Linguagem interativa & $18(78,3)$ & $5(21,7)$ & 1,00 \\
\hline 2.4 Informações corretas & $17(73,9)$ & $6(26,1)$ & 1,00 \\
\hline 2.5 Informações objetivas & $21(91,3)$ & $2(8,7)$ & 1,00 \\
\hline 2.6 Informações esclarecedoras & $22(95,7)$ & $1(4,3)$ & 1,00 \\
\hline 2.7 Informações necessárias & $22(95,7)$ & $1(4,3)$ & 1,00 \\
\hline 2.8 Sequência lógica das ideias & $21(91,3)$ & $2(8,7)$ & 1,00 \\
\hline 2.9 Tema atual & $23(100,0)$ & $0(0,0)$ & 1,00 \\
\hline 2.10 Tamanho do texto adequado & $19(82,6)$ & $4(17,4)$ & 1,00 \\
\hline 3. & Relevância & & \\
\hline 3.1 Estimula o aprendizado & $23(100,0)$ & $0(0,0)$ & 1,00 \\
\hline 3.2 Contribui para o conhecimento na área & $23(100,0)$ & $0(0,0)$ & 1,00 \\
\hline 3.3 Desperta interesse pelo tema & $23(100,0)$ & $0(0,0)$ & 1,00 \\
\hline
\end{tabular}

como desempenhar as atividades de cuidado ao idoso. ${ }^{30}$ Nessa perspectiva, ratifica-se a relevância da existência de recursos educativos, construídos e validados, que possam ser utilizados nas atividades de educação em saúde para este público.

A partir da teoria HBM, foi possível identificar os tópicos necessários para abranger os itens que compuseram o folheto educativo, bem como a maneira que estes deveriam ser organizados. Pessoas mais velhas diagnosticadas com a COVID-19 têm maior probabilidade em apresentar complicações e de evoluírem para óbito. ${ }^{3}$ Embora muitas estratégias visem aumentar a conscientização da população para este fato, a forma como as pessoas, em geral, se apropria da informação, pode ser variável; muitas informações que levam à emoções negativas - a exemplo da tristeza, angústia e medo - podem acentuar pensamentos distorcidos sobre a saúde. ${ }^{33}$
Percebe-se ainda grandes dificuldades no direcionamento adequado da sociedade através de informações, principalmente, quando se trata de um grupo com alta susceptibilidade de contágio. A COVID-19 afeta desproporcionalmente os idosos ${ }^{34}$ e estes podem se tornar altamente vulneráveis à infodemia. A degeneração psicobiológica, característica do processo de envelhecimento, pode acarretar em redução das habilidades cognitivas, conferindo, assim, dificuldades em armazenar informações. ${ }^{35}$ Nesse sentido, esse processo não somente impacta na adesão de medidas preventivas pelo idoso que recebe o cuidado, mas também ao idoso que desempenha o papel de cuidador. Desta forma, identificar e compreender o quanto os idosos são susceptíveis à COVID-19, e o quanto podem se tornar gravemente enfermos pela infecção, pode colaborar para que cuidadores reforcem os comportamentos de prevenção da doença ${ }^{36}$, seja 
em relação ao idoso receptor do cuidado ou mesmo com a realização do autocuidado.

No entanto, à medida que as barreiras percebidas sobre essas ações aumentam, a probabilidade de a pessoa realizar o comportamento de prevenção diminui. Assim, é importante explorar as formas de reduzi-las ou removê-las. ${ }^{36}$ No presente estudo, propõe-se aos cuidadores incentivar chamadas de vídeo com familiares para minimizar a distância; manter uma programação de entretenimento; praticar meditação para aliviar o estresse e fazer leves caminhadas dentro de casa ou quintal. Reconhecer esse momento como uma oportunidade para realização de outros hábitos/atividades é essencial.

Desta forma, o estudo poderá contribuir para o avanço científico na área da saúde ao disponibilizar, para o meio assistencial e acadêmico, um material com enfoque educativo de curta e fácil leitura, validado por especialistas e orientado pelos construtos de um modelo teórico. A possibilidade e potencialidade de contribuir com a translação do conhecimento, ou seja, por ser um material autoexplicativo que favorece o processo de ensinoaprendizagem e pode ser utilizado para informar cuidadores e idosos, inclusive na ausência de profissionais de saúde, também é um dos pontos fundamentais da presente investigação.

Entretanto, este estudo apresenta algumas limitações as quais podem ser elucidadas: o folheto educativo não passou por uma validação com público-alvo. Isso pode ser justificado pelas próprias características impostas pela pandemia, cujos atendimentos e visitas domiciliares estavam suspensas pela secretaria de saúde do município onde o estudo foi realizado e apenas eram realizadas em situações de urgência.

\section{CONCLUSÃO}

O folheto educativo intitulado "Promoção de cuidados ao idoso em época de COVID-19" foi construído a partir da revisão da literatura científica e de protocolos da ANVISA, do MS e da OMS e, também, com fundamentação teórica sob a HBM. Ademais, diante do I-CVI e IVC global de 1,0 para ambos além de teste binomial que confirmou concordância estatisticamente superior a $80 \%$, o folheto foi considerado válido por especialistas. Desse modo, disponibiliza-se um folheto educativo para fornecer informações fundamentais sobre a prevenção da COVID-19 que necessitaram ser introduzidas na rotina do "novo normal" de cuidadores no desempenho das atividades de assistência direta aos idosos.

Espera-se que este folheto contribua na prática de profissionais, docentes e estudantes da área da saúde com o intuito de disseminarem as informações sobre os comportamentos em saúde relacionados à prevenção da COVID-19 nas atividades de ensino, pesquisa e/ou extensão e, consequentemente, colaborar para redução de agravos maiores e mortalidade de idosos.

\section{REFERÊNCIAS}

1. World Health Organization. WHO Coronavirus Disease (COVID-19) Dashboard. World Health Organization. 2021. p. https://covid19.who.int/.

2. Dosa D, Jump RLP, LaPlante K, Gravenstein S. LongTerm Care Facilities and the Coronavirus Epidemic: Practical Guidelines for a Population at Highest Risk. Journal of the American Medical Directors Association. 2020;21(5):569-71.

3. Zhou F, Yu T, Du R, Fan G, Liu Y, Liu Z, et al. Clinical course and risk factors for mortality of adult inpatients with COVID-19 in Wuhan, China: a retrospective cohort study. The Lancet. 2020;395(10229):1054-1062.

4. Brasil. Ministério da Saúde. Secretaria de Atenção à Saúde. Protocolo do Manejo Clínico do Coronavírus (COVID-19) na Atenção Primária. Secretaria de Atenção Primária à Saúde (SAPS) [Internet]. 2020; Available from: http:// docs.bvsalud.org/biblioref/2020/05/1095920/20200504protocolomanejo-ver09.pdf

5. Greenhalgh T, Knight $M, A^{\prime}$ Court $C$, Buxton $M$, Husain L. Management of post-acute covid-19 in primary care. The BMJ. 2020;(370):m3026.

6. Johnston C, Brown ER, Stewart J, Stankiewicz Karita HC, Kissinger PJ, Dwyer J, et al. Hydroxychloroquine with or without azithromycin for treatment of early SARS-CoV-2 infection among high-risk outpatient adults: a randomized clinical trial. EClinicalMedicine. 2021;100773.

7. Horby P, Lim WS, Emberson JR, Mafham M, Bell JL, Linsell $L$, et al. Dexamethasone in hospitalized patients with Covid-19. New England Journal of Medicine. 2021;384(8):693-704.

8. Choudhary R, Sharma AK. Potential use of hydroxychloroquine, ivermectin and azithromycin drugs in fighting COVID-19: trends, scope and relevance. Vol. 35, New Microbes and New Infections. 2020. p. 100684.

9. Kaur H, Shekhar N, Sharma S, Sarma P, Prakash A, Medhi B. Ivermectin as a potential drug for treatment of COVID-19: an in-sync review with clinical and computational attributes. Pharmacological Reports. 2021;1-14.

10. Voysey M, Clemens SAC, Madhi SA, Weckx LY, Folegatti PM, Aley PK, et al. Safety and efficacy of the ChAdOx1 nCoV-19 vaccine (AZD1222) against SARS-CoV-2: an interim analysis of four randomised controlled 
trials in Brazil, South Africa, and the UK. The Lancet. 2021;397(10269):99-111.

11. Agencia Nacional de Vigilância Sanitária. Parecer Público de avaliação de solicitação de autorização temporária de uso emergencial, em caráter experimental, da vacina adsorvida covid-19 (inativada) - Instituto Butantan. Processo: 25351.821027/2021-12 [Internet]. 2021. p. 32 p. Available from: https://www.gov.br/anvisa/ pt-br/assuntos/noticias-anvisa/2021/confira-materiaisda-reuniao-extraordinaria-da-dicol/ppam-final-vacinaadsorvida-covid-19-inativada-butantan.pdf

12. Hodgson SH, Mansatta K, Mallett G, Harris V, Emary KRW, Pollard AJ. What defines an efficacious COVID-19 vaccine? A review of the challenges assessing the clinical efficacy of vaccines against SARS-CoV-2. Vol. 21, The Lancet Infectious Diseases. 2021. p. e26-35.

13. Brasil. Ministério da Saúde. Portaria GM nº 963 de 27 de maio de 2013 que redefine a Atenção Domiciliar no âmbito do Sistema Único de Saúde (SUS) [Internet]. 2013. Available from: http://bvsms.saude.gov.br/bvs/ saudelegis/gm/2013/prt0963_27_05_2013.html

14. Chu DK, Akl EA, Duda S, Solo K, Yaacoub $S$, Schünemann $\mathrm{HJ}$, et al. Physical distancing, face masks, and eye protection to prevent person-to-person transmission of SARS-CoV-2 and COVID-19: a systematic review and meta-analysis. The Lancet. 2020;395(10242):1973-1987.

15. Bavel JJV, Baicker K, Boggio PS, Capraro V, Cichocka A, Cikara $M$, et al. Using social and behavioural science to support COVID-19 pandemic response. Nature Human Behaviour. 2020;4(5):460-71.

16. Oliveira SRG, Wendhausen ALP. (Re)significando a educação em saúde: dificuldades e possibilidades da Estratégia Saúde da Família. Trabalho, Educação e Saúde. 2014;12(1):129-47.

17. Parent K, Jones K, Phillips L, Stojan JN, House JB. Teaching Patient- and Family-Centered Care: Integrating Shared Humanity into Medical Education Curricula. The AMA Journal of Ethic. 2016;18(1):24-32.

18. Cardoso RSS, Sá SPC, Domingos AM, Sabóia VM, Maia TN, Padilha JMFO, et al. Tecnologia educacional: um instrumento dinamizador do cuidado com idosos. Rev Bras Enferm. 2018;71(Suppl 2):786-92.

19. Nascimento ÉA, Tarcia RML, Magalhães LP, Soares MAL, Suriano MLF, Domenico EBL. Folhetos educativos em saúde: estudo de recepção. Revista da Escola de Enfermagem da USP. 2015;49(3):435-42.

20. Echer IC. Elaboração de manuais de orientação para o cuidado em saúde. Revista Latino-Americana de Enfermagem. 2005;13(5):754-7.

21. Maiman LA, Becker MH. The Health Belief Model: Origins and Correlates in Psychological Theory. Health Education \& Behavior. 1974;2(4):336-53.

22. Hoffmann T, Worrall L. Designing effective written health education materials: Considerations for health professionals. Disability and Rehabilitation. 2004;26(9):1166-73.
23. Oliveira Lopes MV, Silva VM, Araujo TL. Methods for establishing the accuracy of clinical indicators in predicting nursing diagnoses. International Journal of Nursing Knowledge. 2012;23(3):134-9.

24. Jasper MA. Expert: a discussion of the implications of the concept as used in nursing. Journal of Advanced Nursing. 1994;20(4):769-76.

25. Leite SS, Áfio ACE, Carvalho LV, Silva JM, Almeida PC, Pagliuca LMF. Construction and validation of an Educational Content Validation Instrument in Health. Revista brasileira de enfermagem. 2018;71(4):1635-41.

26. Polit DF, Beck CT. The content validity index: Are you sure you know what's being reported? Critique and recommendations. Research in Nursing and Health. 2006;29(5):489-97.

27. Ximenes MAM, Fontenele NAO, Bastos IB, Macedo TS, Neto NMG, Caetano JA, et al. Construction and validation of educational booklet content for fall prevention in hospitals. Acta Paulista de Enfermagem. 2019;32(4):433-41.

28. Grudniewicz A, Bhattacharyya O, McKibbon KA, Straus SE. Redesigning printed educational materials for primary care physicians: Design improvements increase usability. Implementation Science. 2015;10:156.

29. Araújo SNM, Santiago RF, Barbosa CNS, Figueiredo MLF, Andrade EMLR, Nery IS. Technologies for care to elderly in health services: an integrative review. Enfermeria Global. 2017;16(2):579-95.

30. Jesus TIM, Orlandi AAS, Zazzetta MS. Sobrecarga, perfil e cuidado: cuidadores de idosos em vulnerabilidade social. Revista Brasileira de Geriatria e Gerontologia. 2018;21(2):199-209.

31. Petrovic M, Gaggioli A. Digital mental health tools for caregivers of older adults - A scoping review. Frontiers in Public Health. 2020;8:128.

32. Diniz MAA, Melo BRS, Neri KH, Casemiro FG, Figueiredo LC, Gaioli CCLO, et al. Estudo comparativo entre cuidadores formais e informais de idosos. Ciência \& Saúde Coletiva. 2018;23(11):3789-98.

33. Faro A, Bahiano MA, Nakano TC, Reis C, Silva BFP, Vitti LS. COVID-19 e saúde mental: a emergência do cuidado. Estudos de Psicologia (Campinas). 2020;37:e200074.

34. Mueller AL, McNamara MS, Sinclair DA. Why does COVID-19 disproportionately affect older people? Aging. 2020;12(10): 9959-9981.

35. Aigbogun MS, Stellhorn R, Krasa H, Kostic D. Severity of memory impairment in the elderly: Association with health care resource use and functional limitations in the United States. Alzheimer's and Dementia: Diagnosis, Assessment and Disease Monitoring. 2017;8:51-9.

36. Carico R, Sheppard J, Thomas CB. Community pharmacists and communication in the time of COVID-19: Applying the health belief model. Research in Social and Administrative Pharmacy. 2020;20:30293-X. 


\section{Contribuições dos autores}

HNG, GHRC e FO: trabalharam na coleta e tabulação dos dados e na redação do artigo; FO, RNRM, DDT: trabalharam na concepção e delineamento do estudo e realizaram a análise dos dados; FO, RNRM, DDA, ACM, SNA e DDT: trabalharam na redação e revisão crítica do manuscrito. Todos os autores leram e aprovaram a versão final enviada.

\section{Fontes de financiamento}

Programa institucional de auxílio ao enfrentamento à pandemia Covid-19, seus impactos e efeitos (PIE-COVID-19)" da UFSJ. Edital

Autor Correspondente:

Danilo Donizetti Trevisan

ddtrevisan@gmail.com

Editor:

Prof. Dr. Felipe Villela Gomes

Recebido: 08/02/2021

Aprovado: 19/05/2021 